\title{
Relationships between Tongue and Lip Movement and Physical Fitness among Independent Community-Dwelling Elderly in Japan
}

\author{
Junko Ouchi, Yuko Hayashi, Michiko Matsubara, Kaoru Fukura, Kumiko Miyata, \\ Michiyo Yamamoto
}

Department of Nursing, Faculty of Health Sciences, Hokkaido University of Science, Sapporo, Japan

Email: ouchi-j@hus.ac.jp

How to cite this paper: Ouchi, J., Hayashi, Y., Matsubara, M., Fukura, K., Miyata, K. and Yamamoto, M. (2020) Relationships between Tongue and Lip Movement and Physical Fitness among Independent Community-Dwelling Elderly in Japan. Advances in Aging Research, 9, 32-44.

https://doi.org/10.4236/aar.2020.92004

Received: January 24, 2020

Accepted: February 25, 2020

Published: February 28, 2020

Copyright (๑) 2020 by author(s) and Scientific Research Publishing Inc. This work is licensed under the Creative Commons Attribution International License (CC BY 4.0).

http://creativecommons.org/licenses/by/4.0/

\begin{abstract}
Aim: The present study aims to examine the association between tongue and lip movement and physical fitness with a sample of independent community-dwelling elderly. Methods: A total of 119 older adults living in a city of Japan participated in this study. Tongue and lip movement was evaluated with oral diadochokinesis (ODK) rate for the syllable "pa", "ta", and "ka" measured by counting syllable repetitions within one second. Physical fitness was objectively evaluated with the Timed-Up and Go Test (TUG) and the 30-Second Chair Stand Test (CS-30). Subjective physical fitness was also assessed with a Physical Component Summary score (PCS) of the 36-item Short-Form Health Survey (SF-36). Partial correlation coefficients between ODK rates and physical fitness measures were obtained after adjusting the effects of age and the number of present teeth. Results: There were significant differences in ODK rates for the syllable "ta" and "ka" between the participants aged 65 - 74 and 75 or older. Significant differences in all the physical fitness measures were also observed in the two age groups. Partial correlation analysis with age and number of present teeth as covariates yielded significant correlations of ODK rates to TUG and PCS, but not with CS-30. Conclusion: Older adults whose functional mobility declined tended to maintain the movement of tongue and lips. Moreover, those who perceived declining physical fitness also tended to show a decline of tongue and lip movement. Thus, the present study added some knowledge on the relationships between general physical fitness and tongue and lip movement. It also made several suggestions for future interventions based on the findings.
\end{abstract}




\section{Keywords}

Tongue and Lip Movement, Oral Diadochokinesis, Physical Fitness, Elderly

\section{Introduction}

The population is rapidly aging in Japan, even though it has attained one of the longest life expectancies in the world. Notably, Japan's healthy life expectancy has not extended similarly to its life expectancy [1]. More endeavors are needed so that the elderly can maintain their functioning and independence.

Recent arguments emphasize the importance of oral function as a critical factor to keep older adults functional and independent [2] [3]. The oral function is essential not only in terms of verbal communication but also concerning the intake of adequate nutrition. Malnutrition leads to sarcopenia or the decline of skeletal muscle tissue and function with age [4]. Because sarcopenia is one of the most significant causes of functional decline and loss of independence in older adults [4], a proper oral function is essential for the elderly to remain functional and independent.

Among various aspects of oral function, such as occlusal force and saliva flow, the present study focused on tongue and lip movement because of its importance in ingesting and processing foods [5]. Research often uses oral diadochokinesis (ODK) to evaluate the dexterity of tongue and lip movement [2] [6]. It requires an individual to repeatedly produce strings of syllables as fast as possible in a certain period, such as five seconds [6]. ODK rate for the individual is obtained by calculating the number of articulated syllables per unit time, such as one second [6]. Syllable "pa", "ta", and "ka" are often utilized for Japanese samples [7]. ODK for the syllable "pa" represents lip movement, whereas ODK for the syllable "ta" and "ka" represents the movement of the dorsum of the tongue [8].

In research conducted in Japan with samples of independent older adults, mean ODK rates range between 5 to 7 per second [6] [7] [8] [9], whereas those ranging 4 to 5 per second in research with samples of older adults needed help for activities of daily living [10] [11]. Research also suggests ODK rate declines with age [6] [7] [8] [9].

In addition to functional decline and aging, general physical fitness may be significantly associated with tongue and lip movement in older adults. Izuno et al. [6] examined associations between ODK and five measures of physical fitness with a sample of 66 independent community-dwelling elderly. In that study, mean ODK rates for syllable "pa", "ta", and "ka" ranged from 6.3 to 6.9 for the participants aged $65-69$, and from 5.2 to 6.3 for those aged 70 or older. They reported significant associations between ODK for the syllable "pa" and functional mobility, ODK for the syllable "ta" and lower body flexibility, and ODK for the syllable "ka" and grip strength as well as balance ability. They concluded 
that tongue and lip movement was associated with physical fitness. However, they suggested that more examinations with larger samples are needed to gain insights on associations between tongue and lip movement and physical fitness.

Watanabe et al. [8] compared the ODK rate among robust, prefrail, and frail older people with a large sample in Japan. The study assigned 4720 community-dwelling elderly into three groups, namely the robust, prefrail, and frail group. The frailty phenotype was decided according to five domains of physical fitness, including mobility, strength, endurance, physical activity, and nutritional status. The results denoted that the frail group had a significantly lower ODK rate than the robust group. The study also examined influences of age on tongue and lip movement by comparing ODK rates among four age groups: 65 - 69, 70 74, $75-79,80$ or older. They reported that ODK rates declined with age, suggesting deteriorations of tongue and lip movement with age. Their findings suggest significant associations between tongue and lip movement and physical fitness. However, they did not directly investigate associations between specific physical fitness measures and tongue and lip movement. Further investigations are, therefore, needed to know what aspects of physical fitness have significant associations with tongue and lip movement.

It is valuable to understand relationships between tongue and lip movement and physical fitness in terms of preventing loss of independence in community-dwelling elderly. Such preventive interventions in Japan frequently include exercise aimed at promoting motor functions contributing to physical fitness [12] [13]. If physical fitness is significantly associated with tongue and lip movement, such interventions may work for promoting oral function as well. Moreover, it becomes easier to identify older adults who may have some problems in eating and swallowing before examination of oral function, provided the associations are significant. Concerning the issue mentioned above, the present study also examined associations between tongue and lip movement and subjective physical fitness to explore the effectiveness of an elderly's perceptions of physical fitness on detecting a decline in tongue and lip movement.

In summary, the present study aimed to examine relationships between tongue and lip movement and physical fitness in independent community-dwelling elderly in Japan.

\section{Methods}

\subsection{Participants}

People aged 65 years or older who lived in a residential area of an urban city in Hokkaido, Japan, were recruited as participants for a survey held on a university campus located in the city in March 2018. They were informed of the survey by flyers circulated through the community centers of that area.

\subsection{Data Collection}

Oral diadochokinesis was measured to assess tongue and lip movement. Partici- 
pants were asked to articulate the "pa" syllable repetitively as quickly as possible for 5 seconds. The number of articulations was counted with an automatic counter (T.K.K.3351 digital counter, Takei Scientific Instruments Co. Itd., Nigata city, Nigata, Japan). The same procedure was repeated for syllables "ta" and "ka". The average number of articulations per second for "pa", "ta", and "ka" were respectively calculated as the ODK rate for each syllable.

Objective physical fitness was evaluated in terms of lower limb strength and functional mobility. Lower limb strength was assessed with a 30-Second Chair Stand Test (CS-30) [14]. The test was started at the posture of participants sitting on the chair with both limbs spread to the width of the shoulder, separated the back from the backrest, and the arms were assembled in front of the chest. The participants were then asked to rise and sit on a chair repeatedly as quickly as possible within 30 seconds. The number of times the participant stood up for 30 seconds was counted as his/her CS-30 score. According to Nakatani et al. [14], standard scores for male adults aged $65-69,70-74,75-79,80$, and older are respectively $18-21,16-20,15-17$, and $14-16$. Likewise, those for female adults are $17-21,15-19,13-17$, and 13 - 16. Functional mobility, which incorporates limb muscle strength, walking ability, dynamic balance, and agility, was evaluated with Timed-Up and Go Test (TUG) [15]. TUG measures the time that takes a participant to stand up from a chair, walk a distance of 3 meters, turn, walk back to the chair, and sit down. The shorter the time of TUG, the better the functional mobility.

Subjective physical fitness was evaluated with the Physical Component Summary (PCS) score of the Japanese version of Medical Outcomes Study 36-item Short Form Survey version 2.0 (SF-36v2) [16]. The scale is widely used to evaluate health-related quality of life. It consists of eight subscales: physical functioning, physical role functioning, bodily pain, general health perception, vitality, social role functioning, emotional role functioning, and mental health. Each subscale score can range from 0 to 100. A higher score means a higher level of health-related QOL. In this study, the PCS score was used to assess participants' subjective physical fitness. It was calculated by an algorithm provided by iHope International Corporation after raw scores of the subscales were standardized using means and standard deviations from the general Japanese population provided by the same company. The mean PCS for people aged 60 - 69 and $70-79$ gained from the general Japanese population are respectively $46.9 \pm 11.6$ and $42.1 \pm 14.6$ regardless of sex [17].

Participant demographic information including age, gender, family structure, form of living, and regular hospital visit was collected with a self-administrated questionnaire. Participants were also asked about the number of outings per week as an indicator of their activity. Their level of instrumental activities of daily living (IADL) was assessed as an indicator of physical, cognitive, and social function by the Rouken-Shiki scale [18]. This 13-item scale was developed in Japan and ranged from 0 to 13 , with a lower score indicating poorer IADL. Nutri- 
tional status was evaluated with body mass index (BMI) calculated based on measured weight and height. Numbers of both present and functional teeth were also counted in an oral examination by dental hygienists as an index of oral condition. Existing teeth are the natural teeth retained, while functional teeth are both natural and artificial ones that are present.

\subsection{Data Analysis}

All data were statistically analyzed with SPSS statistics 24 (IBM, Armonk, NY, USA). First, to explore age differences in ODK rate and physical fitness measures, participants were divided into two groups: $65-74$ ( $\leq 74$ age group) and 75 years or older ( $\geq 75$ age group). Such age classification was thought to be meaningful because healthcare and welfare policies for the elderly in Japan are developed based on those age groups. Then, influences of age and sex on ODK rates and physical fitness measures were examined by two-way analysis of variance (ANOVA). Sidak test was conducted as a post-hoc test when the main effects of either age or sex were significant. Finally, partial correlation coefficients were obtained to examine relations between ODK rates and physical fitness measures independent of covariates: age and number of present teeth.

\subsection{Ethical Consideration}

Approval of the protocol was obtained from the Institutional Review Board at the authors' affiliated university (IRB No. 206) in February 2017. Verbal and written informed consents were obtained from individual participants.

\section{Results}

\subsection{Participant Characteristics}

One hundred nineteen older adults participated in this survey. Participant characteristics are shown in Table 1. The participants' age ranged from 65 to 88 years old. More than half of the participants were female, and $70 \%$ of participants' households were only for the elderly. Nearly $80 \%$ of the participants owned their houses or apartment rooms. Besides, over $90 \%$ went outside more than 3 times a week. More than $80 \%$ of the participants visit clinic(s) or hospital(s) regularly. BMI as nutritional status ranged from 15.1 - 32.3, with its mean 23.5 ( \pm 3.4$)$. The number of present teeth of the participants ranged from 0 to 31 , whereas the number of functional teeth ranged from 23 to 32 .

\subsection{Oral Diadochokinesis}

Means and standard deviations of the ODK rate for the three syllables in all, female, and male participants in the two age groups are shown in Table 2. Data on the ODK rate was almost normally distributed. There was no sex difference in means of ODK rate for the syllable "pa" $(P=0.41)$, "ta" $(P=0.98)$, and "ka" $(P=$ $0.50)$. On the other hand, means of ODK rate for the syllable "ta" $(P=0.01)$ and "ka" $(P=0.01)$ in the $\leq 74$ age group was higher than the $\geq 75$ age group. 
Table 1. Participants' characteristics $(n=119)$.

\begin{tabular}{|c|c|c|c|c|}
\hline Characteristic & $M$ & $S D$ & $n$ & $\%$ \\
\hline Age & 73.3 & 5.3 & 119 & \\
\hline \multicolumn{5}{|l|}{ Gender } \\
\hline Male & & & 53 & 44.5 \\
\hline Female & & & 66 & 55.5 \\
\hline \multicolumn{5}{|l|}{ Family structure } \\
\hline Living alone & & & 18 & 15.1 \\
\hline Living with suppose & & & 65 & 54.6 \\
\hline Living with child(ren) & & & 33 & 27.7 \\
\hline Others & & & 3 & 2.5 \\
\hline \multicolumn{5}{|l|}{ Form of living } \\
\hline Own house/apartment rooms & & & 98 & 82.3 \\
\hline Rented house/apartment rooms & & & 14 & 11.8 \\
\hline Others & & & 7 & 5.9 \\
\hline \multicolumn{3}{|c|}{ Number of those who visit clinic(s)/hospital(s) regularly } & 99 & 83.2 \\
\hline \multicolumn{5}{|l|}{ Number of outing per week } \\
\hline $1-2$ & & & 12 & 10.1 \\
\hline $3-4$ & & & 50 & 42.0 \\
\hline $5-6$ & & & 29 & 24.4 \\
\hline Everyday & & & 28 & 23.5 \\
\hline IADL & 12.1 & 1.2 & & \\
\hline BMI & 23.5 & 3.4 & & \\
\hline Number of present teeth & 20.0 & 8.0 & & \\
\hline Number of functional teeth & 27.6 & 1.5 & & \\
\hline
\end{tabular}

Note. M, mean; SD, standard deviation; IADL, instrumental activities of daily living; BMI, body mass index.

Table 2. Means and standard deviations of oral diadochokinesis rates and physical fitness variables for all, female, and male participants in two age groups $(\mathrm{n}=119)$.

\begin{tabular}{|c|c|c|c|c|c|c|c|c|c|c|c|c|}
\hline \multirow[b]{3}{*}{ Variable } & \multicolumn{6}{|c|}{65 - 74 years } & \multicolumn{6}{|c|}{75 years or older } \\
\hline & \multicolumn{2}{|c|}{ All } & \multicolumn{2}{|c|}{ Female } & \multicolumn{2}{|c|}{ Male } & \multicolumn{2}{|l|}{ All } & \multicolumn{2}{|c|}{ Female } & \multicolumn{2}{|c|}{ Male } \\
\hline & $M(S D)$ & $n$ & $M(S D)$ & $n$ & $M(S D)$ & $n$ & $M(S D)$ & $n$ & $M(S D)$ & $n$ & $M(S D)$ & $n$ \\
\hline ODK “pa” & $6.1(0.9)$ & 67 & $6.3(0.8)$ & 43 & $5.9(1.0)$ & 24 & $5.9(0.8)$ & 44 & $5.8(0.9)$ & 18 & $5.9(0.7)$ & 26 \\
\hline ODK “ta” & $6.2(0.9)$ & 66 & $6.3(0.9)$ & 42 & $5.9(1.0)$ & 24 & $5.8(0.8)$ & 44 & $5.7(0.9)$ & 18 & $5.8(0.8)$ & 24 \\
\hline ODK “ka” & $5.9(0.9)$ & 67 & $6.0(0.9)$ & 43 & $5.8(1.0)$ & 24 & $5.5(0.7)$ & 45 & $5.5(0.8)$ & 19 & $5.5(0.6)$ & 26 \\
\hline CS-30 & $20.9(5.8)$ & 68 & $21.4(5.5)$ & 43 & $20.1(6.3)$ & 25 & $17.9(5.9)$ & 46 & $17.7(6.9)$ & 20 & $18.1(5.2)$ & 26 \\
\hline TUG (sec) & $6.8(1.9)$ & 68 & $6.8(2.0)$ & 43 & $6.9(1.8)$ & 25 & $7.9(1.5)$ & 45 & $8.0(1.5)$ & 20 & $7.8(1.5)$ & 25 \\
\hline PCS & $47.0(10.0)$ & 67 & $48.8(6.8)$ & 43 & $48.7(10.4)$ & 24 & $44.1(11.9)$ & 45 & $43.1(12.4)$ & 20 & $44.9(11.6)$ & 25 \\
\hline
\end{tabular}

Note. M, mean; SD, standard deviation; ODK, oral diadochokinesis; CS-30, the 30-Second Chair Stand Test; TUG, Timed-Up \& Go test; PCS, Physical Component Summary score of Medical Outcomes Study 36-item Short-Form Survey. 
However, no significant difference was found in the ODK rate for the syllable "pa" between the age groups $(P=0.12)$.

\subsection{Physical Fitness}

Table 2 also presents means and standard deviations of the physical fitness measures for all, female, and male participants in the two age groups. Data of each physical fitness measure was also almost normally distributed. Between female and male participants, there were no significant differences in mean scores of all the measures: CS-30 $(P=0.70)$; TUG $(P=0.83)$; PCS $(P=0.66)$. On the other hand, significant age differences in means were observed in all the measures. The mean of CS-30 in the $\leq 74$ age group was significantly larger than the $\geq 75$ age group $(P=0.01)$. Similarly, the $\leq 74$ age group has a shorter mean TUG time compared with the $\geq 75$ age group $(P=0.002)$. In addition to these objective physical fitness measures, a mean PCS score for the $\leq 74$ age group was significantly higher than one for the $\geq 75$ age group $(P=0.02)$.

\subsection{Associations between ODK and Physical Fitness}

Prior to partial correlation analysis, intercorrelations for participant characteristics, ODK rate, and physical fitness measures were explored (Table 3 ). As the table shows, age was significantly associated with ODK rate for the syllable "ta" $(P=0.004)$ and "ka" $(P=0.014)$ as well as all the physical fitness measures: CS-30 $(P=0.004)$; TUG $(P=0.001)$; PCS $(P=0.01)$. However, it was not related to the ODK rate for the syllable "pa" $(P=0.15)$. In addition to age, the number of present teeth had significant associations with ODK rate for the syllable "ta" $(P=0.001)$ and "ka" $(P=0.001)$ but not with that for the syllable "pa" $(P=0.07)$. Number of present teeth was also significantly associated with CS-30 $(P=0.002)$ and TUG $(P=0.008)$ but not with PCS $(P=0.94)$. Gender was associated only

Table 3. Intercorrelations for participant characteristics, oral diadochokinesis rates, and physical fitness measures $(n=102)$.

\begin{tabular}{|c|c|c|c|c|c|c|c|c|c|c|}
\hline Variable & 1 & 2 & 3 & 4 & 5 & 6 & 7 & 8 & 9 & 10 \\
\hline 1. Age & - & & & & & & & & & \\
\hline 2. Gender & $-0.33^{* *}$ & - & & & & & & & & \\
\hline 3. BMI & $0.05^{* *}$ & $-0.20^{* *}$ & - & & & & & & & \\
\hline 4. Teeth & $-0.28^{* *}$ & 0.16 & $-0.10^{* *}$ & - & & & & & & \\
\hline 5. ODK "pa" & $-0.14^{*}$ & $0.17^{*}$ & $-0.12^{* *}$ & $0.18^{*}$ & - & & & & & \\
\hline 6. ODK “ta" & $-0.28^{* *}$ & $0.14^{*}$ & $-0.18^{*}$ & $0.31^{* *}$ & $-0.78^{* *}$ & - & & & & \\
\hline 7. ODK "ka" & $-0.24^{* *}$ & $0.20^{*}$ & $-0.15^{* *}$ & $0.31^{* *}$ & $-0.73^{* *}$ & $0.87^{* *}$ & - & & & \\
\hline 8. CS-30 & $-0.29^{* *}$ & 0.16 & $-0.25^{* *}$ & $0.30^{* *}$ & $-0.25^{* *}$ & $0.24^{* *}$ & $0.20^{* *}$ & - & & \\
\hline 9. TUG & $0.33^{* *}$ & $-0.12^{*}$ & $0.21^{*}$ & $-0.23^{*}$ & $-0.25^{* *}$ & $-0.34^{* *}$ & $-0.28^{* *}$ & $-0.31^{* *}$ & - & \\
\hline 10. PCS & $-0.26^{* *}$ & $0.06^{*}$ & $-0.33^{* *}$ & $0.01^{*}$ & $-0.32^{* *}$ & $0.38^{* *}$ & $0.32^{* *}$ & $0.29^{* *}$ & $-0.38^{* *}$ & - \\
\hline
\end{tabular}

Note. BMI, Body mass index; Teeth; the number of the present teeth; ODK, oral diadochokinesis; CS-30, the 30-Second Chair Stand Test; TUG, Timed-Up \& Go test; PCS, Physical Component Summary score of Medical Outcomes Study 36 -item Short-Form Survey. ${ }^{*} P<0.05$. ${ }^{* *} P<0.01$. 
with the ODK for the syllable "ka" weakly but not with the ODK for the syllable "pa" $(P=0.08)$ and "ka" $(P=0.16)$ as well as any of the physical fitness measures: CS-30 $(P=0.12)$; TUG $(P=0.24)$; PCS $(P=0.52)$.

Based on the results above, partial correlation coefficients between ODK rate and physical fitness measures were obtained with age and the number of present teeth as covariates (Table 4). As it illustrates, no significant association was found between CS-30 and ODK rate for all the syllables: "pa" $(P=0.05)$; "ta" ( $P$ $=0.22)$; "ka" $(P=0.39)$. TUG had a significant but weak association with ODK rate for the syllable "pa" $(P=0.048)$ and "ta" $(P=0.02)$ but not with ODK rate for syllable "ka" $(P=0.06)$. On the other hand, PCS had significant associations with ODK rate for all the syllables: "pa" $(P=0.001)$; "ta" $(P<0.001)$; "ka" $(P=$ 0.002).

\section{Discussion}

The present study investigated associations between tongue and lip movement and physical fitness with a sample of community-dwelling independent older adults. Results suggested that tongue and lip movement was significantly associated with functional mobility and subjective physical fitness even after adjusting the effects of age and number of present teeth.

Participants in the present study could be characterized as being generally healthy, functional, and active even most of them visit clinic(s) or hospital(s) regularly. Results of BMI, as well as the number of present and functional teeth, indicated that they generally stayed in good nutritional status and oral condition. The mean score of the IADL scale in the present study was close to its maximum score, suggesting they were adequately functional to live independently. Also, most participants regularly outed more than 3 times a week, and nearly half of them outed more than 5 times a week, suggesting that they had a relatively active lifestyle.

Results also suggested that participants in the present study were average in physical fitness. Results of CS-30 indicated that the participants had average lower limb strength based on standard scores proposed by Nakatani et al. [14].

Table 4. Partial correlations for oral diadochokinesis and physical fitness measures with age and number of present teeth as covariates $(n=96)$.

\begin{tabular}{lccccc}
\hline Variable & 1 & 2 & 3 & 4 & 5 \\
\hline 1. ODK "pa" & - & & & & \\
2. ODK "ta" & $0.77^{* *}$ & - & & \\
3. ODK "ka" & $0.72^{* *}$ & $0.86^{* *}$ & - & - & - \\
4. CS-30 & $0.19^{*}$ & $0.12^{* *}$ & 0.09 & -0.21 & $-0.34^{* *}$ \\
5. TUG & $-0.19^{* *}$ & $-0.24^{* *}$ & -0.19 & $0.26^{* *}$ & - \\
6. PCS & $-0.31^{* *}$ & $0.36^{* *}$ & $-0.30^{* *}$ & \\
\hline
\end{tabular}

Note. ODK, oral diadochokinesis; CS-30, the 30-Second Chair Stand Test; TUG, Timed-Up \& Go test; PCS, Physical Component Summary score of Medical Outcomes Study 36 -item Short-Form Survey. ${ }^{*} P<0.05 .{ }^{* *} P<0.01$. 
Results of TUG were also similar to results from past literature [19], suggesting that the participants had average functional mobility compared to their counterparts. On the other hand, the results of PCS suggested that participants in the present study had a slightly higher level of subjective physical fitness compared with the reference scores that Fukuhara and Suzukamo [17] presented, even though age classification was different between the studies. Thus, the participants in this study were thought to be generally and physically - healthy and functional.

Mean ODK rates in the present study were similar to results from past studies [7] [8]. Results on the associations between age and ODK for the syllable "ta" and "ka" indicated that tongue movement declines with age among independent older adults. This deterioration is consistent with findings from past studies examining the influences of age on oral function [6] [7] [8]. Such deteriorations in tongue movement may be related to decreases in tongue thickness and muscle strength with age observed in previous studies [20] [21].

In contrast, ODK for syllable "pa", which represents lip movement, was not affected by age in the present study. This finding is inconsistent with results from past literature reporting that ODK for the syllable "pa" also declined with age [6] [7] [8]. This decline may be due to the distinctions in age classifications between the studies. For example, Watanabe et al. [8] classified participants into four age groups and observed differences between them, while the present study divided those into two. It was believed that a smaller age classification would make it easier to detect age-dependent effects. Another possible reason for the inconsistency is that lip movement is less likely to be affected by age compared to tongue movement. In the present study, the correlation analysis yielded no significant linear association between age and ODK rate for the syllable "pa". This result is consistent with findings from Watanabe et al. [8]. In their study, both of the $70-74$ and $75-79$ age groups had the same means and standard deviations of ODK for the syllable "pa". Both findings indicated no linear association between age and ODK for the syllable "pa", suggesting that lip movement can be stable while people are getting old. Past literature has rarely focused on the difference in age-dependent changes between tongue and lip movement. Further examinations are required to address this issue adequately.

In addition to age, the number of present teeth was significantly associated with ODK rates for all the syllables in the present study. The number of present teeth is a cardinal indicator of oral health condition [22]. Eklund and Burt [22] also reported that oral health was affected by the patients' cumulative disease experience and treatments chosen. The findings indicate that maintaining good oral and general health contributes to a higher number of present teeth as well as better tongue and lip movement.

Results of the partial correlation analysis yielded significant associations between some of the physical fitness measures and ODK rates even after adjusting the effects of age and number of present teeth. Among the measures, PCS, which 
represents subjective physical fitness, significantly correlated to ODK rates for all the syllables. The correlation coefficients for those associations indicate that the PCS data can explain approximately $10 \%$ of the variance of the ODK rate data in the present study. This finding suggests that when the elderly perceive a decline in general physical fitness, their actual tongue and lip movements also deteriorate. This degeneration is practically relevant because the elderly's subjective evaluation of physical fitness helps healthcare professionals identify the high-risk group for declining movements of the tongue and lips without objectively evaluating them. It may also be helpful for older adults to be timely aware of their declining tongue and lip movement by themselves. Such early awareness can lead to an early intervention aimed at promoting oral function, including tongue and lip movement, which in turn, contribute to delaying new onsets of sarcopenia and physical frailty [3]. In the present study, PCS of SF-36 was utilized to assess physical fitness subjectively. Future studies should explore whether there are other valid and straightforward measures of subjective physical fitness that are associated with tongue and lip movement.

Despite the significant associations between ODK rate and PCS, the objective physical fitness measures had no or only weak associations with ODK rates. Scores of CS-30 were not significantly associated with ODK for any of the syllables, suggesting no direct association between lower limb strength and tongue and lip movement. Scores of TUG, on the other hand, had a weak but significant association with ODK rate for the syllable "pa" and "ta", suggesting that functional mobility is weakly associated with lip and tongue body movement. Such a distinction was possibly observed because ODK evaluates tongue and lip movement in terms of dexterity rather than muscle strength. TUG is a more comprehensive measure of motor functions compared with CS-30 that represents mainly lower limb strength. This disparity may have contributed to the significant association between ODK and TUG.

The findings also can be applied to efforts to retain and improve the elderly's tongue and lip movement. Several studies have shown the effectiveness of exercise programs for community-dwelling elderly on the improvement of physical functions including muscle strength as well as functional mobility with a sample of community-dwelling elderly in Japan [23] [24]. Such effective exercise programs may be able to show their effectiveness in promoting tongue and lip movement if the functional ability is significantly associated with it. Such application of exercise programs to promote the oral function in older adults is vital in terms of cost and resource-saving for long-term care prevention efforts in super-aged societies, such as Japan. It would also be efficient for older people, as they do not need to attend multiple intervention programs.

Thus, the results of the present study suggest that tongue and lip movement is associated with physical fitness. Some suggestions were also discussed based on the findings for future interventions targeting independent community-dwelling elderly. However, there is still a dearth of knowledge on relationships between 
tongue and lip movement and physical fitness. For example, research has not adequately examined relationships between physical fitness and other aspects of tongue and lip movement, such as the strength of tongue and orbicularis muscle. Moreover, whether the findings can be applied to those with loss of independent and/or hospitalized ones because the participant in the present study was independent and relatively healthy. Also, it is still unknown whether interventions aimed at promoting motor functions facilitate tongue and lip movement. Therefore, future studies should have a longitudinal research design with multiple measures of tongue and lip movement with both samples of older adults with different functional and health levels.

\section{Conclusion}

The present study found that tongue and lip movement was associated with functional mobility and subjective physical fitness in independent community-dwelling elderly. It was also suggested that when older adults perceived a decline in physical fitness, their tongue and lip movement also tended to decline. This decline may contribute to an early awareness of declining tongue and lip movement by healthcare professionals as well as older adults by themselves. Moreover, effective exercise programs aimed at promoting motor functions may work for retaining tongue and lip movement in the elderly.

\section{Acknowledgements}

The authors are grateful to all participants and volunteers who significantly contributed to this study. Financial supports were provided by the Institute of Research Life Style and Environment for Future, Hokkaido University of Science.

\section{Conflicts of Interest}

No conflict of interest has been declared by the authors.

\section{References}

[1] Ministry of Health, Labour and Welfare (2014) Annual Report on Health, Labor and Welfare 2014. https://www.mhlw.go.jp/wp/hakusyo/kousei/14/dl/1-02-1.pdf

[2] Japanese Society of Gerodontology Academic Committee (2016) Deterioration of Oral Function in the Elderly: The Position Paper from Japanese Society of Gerodontology in 2016. Japanese Journal of Gerodontology, 31, 81-99. (In Japanese)

[3] Tanaka, T., Takahashi, K., Hirano, H., et al. (2017) Oral Frailty as a Risk Factor for Physical Frailty and Mortality in Community-Dwelling Elderly. The Journals of Gerontology: Series A, 73, 1661-1667. https://doi.org/10.1093/gerona/glx225

[4] Cruz-Jentoft, A.J., Baeyens, J.P., Bauer, J.M., et al. (2010) Sarcopenia: European Consensus on Definition and Diagnosis: Report of the European Working Group on Sarcopenia in Older People. Age and Ageing, 39, 412-423. https://doi.org/10.1093/ageing/afq034

[5] Matsuo, K. and Palmer, J.B. (2008) Anatomy and Physiology of Feeding and Swallowing-Normal and Abnormal. Physical Medicine and Rehabilitation Clinics of North America, 19, 691-707. https://doi.org/10.1016/j.pmr.2008.06.001 
[6] Izuno, H., Hori, K., Sawada, M., et al. (2016) Physical Fitness and Oral Function in Community-Dwelling Older People: A Pilot Study. Gerodontology, 33, 470-479. https://doi.org/10.1111/ger.12186

[7] Hara, S., Miura, H. and Yamasaki, K. (2013) Oral Diadochokinesis among Japanese Aged over 55 Years: Analysis of Standard Values. Japanese Journal of Geriatrics, 50, 258-263. (In Japanese) https://doi.org/10.3143/geriatrics.50.258

[8] Watanabe, Y., Hirano, H., Arai, H., et al. (2017) Relationship between Frailty and Oral Function in Community-Dwelling Elderly Adults. Journal of the American Geriatrics Society, 65, 66-76. https://doi.org/10.1111/jgs.14355

[9] Ouchi, J., Hayashi, Y., Matsubara M., et al. (2016) Oral Functions and Nutritional Status among Participants of a Physical Fitness Survey for Community-Dwelling Older Adults in Sapporo. Bulletin of Hokkaido University of Science, 41, 85-90. (In Japanese)

[10] Hara, S., Miura, H., Yamasaki, K. and Sumi, Y. (2012) Association between Activities of Daily Living and Oral Diadochokinesis among Japanese Elderly Individuals in a Nursing Home. Japanese Journal of Gerodontolog, 49, 330-335. (In Japanese) https://doi.org/10.3143/geriatrics.49.330

[11] Ohara, Y., Takagi, D., Edahiro, A., Morishita, S., Watanabe, Y. and Hirano, H. (2015) Relationship between Severity of Dementia and Oral Health Status, Nutritional Status among Older People with Dementia in Group Homes. The Journal of Japan Society for Dental Hygiene, 9, 69-79. (In Japanese)

[12] Kono, A., Kanagawa, K., Ban, M., Kitayama, Y. and Matubara, E. (2002) Evaluation of a Community-Based Preventive Care Program for Elderly Living at Home. Japanese Journal of Public Health, 49, 983-991. (In Japanese)

[13] Ito, Y., Suganuma, K., Serida, T., Sakakibara, R., Chinen, S. and Maruyama, H. (2010) Effects of a Care Prevention Course on Health-Related QOL and Motor Function According to History of Falls. Rigakuryoho Kagaku, 25, 779-784. (In Japanese) https://doi.org/10.1589/rika.25.779

[14] Nakatani, T., Nadamoto, M., Miura K. and Itoh, M. (2002) Validation of a 30-sec Chair-Stand Test for Evaluating Lower Extremity Muscle Strength in Japanese Elderly Adults. Japan Journal of Physical Education, Health, and Sport Sciences, 47, 451-461. (In Japanese) https://doi.org/10.5432/jjpehss.KJ00003390725

[15] Podsiadlo, D. and Richardson, S. (1991) The Timed "Up and Go": A Test of Basic Functional Mobility for Frail Elderly Persons. Journal of the American Geriatrics Society, 39, 142-148. https://doi.org/10.1111/j.1532-5415.1991.tb01616.x

[16] Fukuhara, S., Bito, S., Green, J., Hsiao, A. and Kurokawa, K. (1998) Translation, Adaptation, and Validation of the SF-36 Health Survey for Use in Japan. Journal of Clinical Epidemiology, 51, 1037-1044. https://doi.org/10.1016/S0895-4356(98)00095-X

[17] Fukuhara S. and Suzukamo, Y. (2015) Manual of SF-36v2 Japanese Version. 3rd Edition, iHope International Inc., Kyoto, 170-172.

[18] Koyano, W., Shibata, H., Nakazato, K., Haga, H., Suyama, Y. and Matsuzaki, T. (1989) Mortality in Relation to Instrumental Activities of Daily Living: One-Year Follow-Up in a Japanese Urban Community. Journal of Gerontology, 44, S107-S109. (In Japanese) https://doi.org/10.1093/geronj/44.3.S107

[19] Arai, T., Fujita, H., Hosoi, T., Morita, T. and Ishibashi, H. (2011) Toe Flexor Muscle Strength as a Measure of Motor Function in Community-Dwelling Elderly People. Physical Therapy Japan, 38, 489-496. (In Japanese)

[20] Tamura, F., Kikutani, T., Tohara, T., Yoshida, M. and Yaegaki, K. (2012) Tongue 
Thickness Relates to Nutritional Status in the Elderly. Dysphagia, 27, 556-561. https://doi.org/10.1007/s00455-012-9407-Z

[21] Utanohara, Y., Hayashi, R., Yoshikawa, M., Yoshida, M., Tsuga, K. and Akagawa, Y. (2008) Standard Values of Maximum Tongue Pressure Taken Using Newly Developed Disposable Tongue Pressure Measurement Device. Dysphagia, 23, Article No. 286. https://doi.org/10.1007/s00455-007-9142-Z

[22] Eklund, S. A. and Burt, B.A. (1994) Risk Factors for Total Tooth Loss in the United States; Longitudinal Analysis of National Data. Journal of Public Health Dentistry, 54, 5-14. https://doi.org/10.1111/j.1752-7325.1994.tb01173.x

[23] Arai, T., Obuchi, S., Kojima, M., Matumoto, Y. and Inaba, Y. (2006) The Evaluation of the Relationship between Physical Factors and Effects of Exercise Intervention on Physical Functions in Community-Dwelling Older People. Japanese Journal of Geriatrics, 43, 781-788. (In Japanese) https://doi.org/10.3143/geriatrics.43.781

[24] Hashidate, H., Shimada, H., Shiomi, T. and Sasamoto, N. (2012) The Effects of Combined Functional Training with Muscle Strengthening on Everyday Function in Community-Dwelling Elderly Individuals. Physical Therapy Japan, 39, 159-166. (In Japanese) 